\title{
Associações de peixes na região costeira de Itaipu, Niterói, RJ
}

\author{
Cassiano Monteiro-Neto ${ }^{1,2}$, Rafael A. Tubino ${ }^{1,2}$, Luiz E. S. Moraes ${ }^{1}$, José P. de Mendonça Neto ${ }^{1,2}$, \\ Gustavo V. Esteves ${ }^{2} \&$ Wagner L. Fortes ${ }^{1,2}$
}

1. Laboratório de Biologia do Nécton e Ecologia Pesqueira, Departamento de Biologia Marinha, Universidade Federal Fluminense. Caixa Postal 100644, 24001-970 Niterói, RJ.

2. Programa de Pós-Graduação em Biologia Marinha. Departamento de Biologia Marinha, Universidade Federal Fluminense. (monteiro@vm.uff.br)

\begin{abstract}
Associations of fishes in the coastal region of Itaipu, Niterói. The coastal region of Itaipu, Rio de Janeiro forms a partially sheltered sound, protected by three islands, but still maintaining full communication with the open sea. The sound also receives fresh water input from the Itaipu-Piratininga lagoon system, constituting an area of intense artisanal fisheries. Using data from monthly monitoring of the artisanal fisheries (beach seining, gill netting and hand lining), experimental surf zone beach seine surveys, underwater visual census (Menina, Mãe, and Pai islands), and a multisampling experiment at Itaipu Lagoon, we elaborated a list of fish species occurring in the region, observing their connectivity with different local habitats, including the sound, rocky reefs, the surf zone and Itaipu Lagoon. We identified 183 species including 26 Elasmobranchii, grouped in 13 families, and 157 Actinopterygii grouped in 63 families. The artisanal beach seining caught the greatest number of species (112), followed by the gill netting (94) and hand lining (35). The surf zone beach seining ("picaré") yielded 49 species ( 8 exclusive) represented mostly by juvenile fish and species of little economic importance. Visual censuses resulted in 41 species identified, with 21 exclusive and most of them cryptic. Within the Itaipu lagoon, 46 species were recorded, 18 exclusive and of occasional occurrence. Cluster analysis including 106 non exclusive species resulted in the formation of eight different groups. Groups A and B were composed by species captured exclusively by the artisanal fisheries within the sound, and included species of commercial interest. Group C included species occurring in the fisheries whose juveniles are found in the surf zone. Group D classified species of common occurrence in the fisheries and the islands. Group E was represented by species shared by the fisheries and Itaipu lagoon (mullets, mojarras, herrings). Species from group $\mathrm{F}$ were absent in the islands, those from group $\mathrm{G}$ in the lagoon, and those from group $\mathrm{H}$ were common to all areas considered. Compared with other coastal areas in southeastern Brazil, Itaipu represents an important concentration area for fish biological diversity and biomass, yielding species associations and connectivity between the different local habitats.
\end{abstract}

KEYWORDS. Itaipu, species list, fishes, artisanal fisheries, habitat connectivity.

RESUMO. A região costeira de Itaipu, Rio de Janeiro é guarnecida por três ilhas, formando uma enseada semi-abrigada porém com ampla comunicação com o mar. Esta enseada recebe o aporte de águas do complexo lagunar Itaipu-Piratininga, além da influência de massas d'água oceânicas, constituindo uma área de intensa atividade pesqueira artesanal. Utilizando-se dados provenientes do monitoramento mensal da pesca artesanal (arrastos-de-praia, redes de emalhe e linha de mão), de arrastos experimentais em zona de arrebentação, censos visuais sub-aquáticos (ilhas da Menina, Mãe e Pai) e um experimento multiamostral na lagoa de Itaipu, elaborou-se uma lista de espécies de peixes que ocorrem na região, observando-se as suas conectividades e afinidades aos diversos habitats locais, incluindo a enseada, os recifes rochosos, a zona de arrebentação e a lagoa de Itaipu. Foram identificadas 183 espécies, sendo 26 Elasmobranchii, agrupados em 13 famílias e 157 espécies de Actinopterygii (63 famílias). A pesca de arrasto-de-praia capturou o maior número de espécies (112), seguida das redes de emalhe (94) e da linha de mão (35). Os arrastos na zona de arrebentação (picaré), capturaram apenas 49 espécies (oito exclusivas), principalmente juvenis e sem importância comercial. Os censos visuais identificaram um total de 41 espécies, sendo 21 exclusivas e de caráter críptico. No interior da lagoa de Itaipu foram registradas 46 espécies, 18 exclusivas de caráter ocasional. A análise de agrupamento, incluindo 106 espécies de ocorrência não exclusiva, resultou na formação de oito grupos. Os grupos A e B foram constituídos por espécies associadas exclusivamente às atividades de pesca na enseada, incluindo algumas espécies de interesse comercial. $\mathrm{O}$ grupo $\mathrm{C}$ reuniu as espécies comuns às pescarias cujos juvenis ocorrem na zona de arrebentação. $\mathrm{O}$ grupo $\mathrm{D}$ classificou espécies de ocorrência comum à pesca e às ilhas. $\mathrm{O}$ grupo $\mathrm{E}$ foi representado por espécies associadas à pesca e ocorrentes na lagoa (mugilídeos, gerreídeos e clupeídeos); o grupo F, por espécies ausentes apenas nas ilhas; o grupo G, por espécies ausentes apenas na lagoa e o grupo H, por espécies comuns a todas as áreas amostradas. Comparativamente a outras áreas do sudeste brasileiro, a região costeira de Itaipu representa uma importante área de agregação de diversidade e biomassa da ictiofauna, possibilitando a formação de associações de espécies e a conectividade entre os diferentes habitats locais.

PALAVRAS-CHAVE. Itaipu, lista de espécies, peixes, pesca artesanal, conectividade de habitats.

As zonas costeiras são áreas de transição ecológica que desempenham um importante papel de ligação entre ecossistemas terrestres e marinhos, possibilitando trocas genéticas e de biomassa, caracterizando-as como ambientes dinâmicos e biologicamente diversificados. Habitats costeiros estão expostos a processos marinhos e terrestres, que por sua vez influenciam nas suas características estruturais, afetando em última instância os padrões de distribuição das comunidades de peixes (Nero \& SeAley, 2006). As elevadas concentrações de nutrientes, a presença de gradientes termo-halinos e o fornecimento de abrigo para reprodução, além do suporte à alimentação inicial de um grande número de espécies, são alguns dos fatores que atraem para as zonas costeiras a atenção quanto à conservação de recursos naturais e a manutenção da biodiversidade (LEvinTon, 1982; Livingston, 2002).

Muitas espécies marinhas utilizam diferentes habitats costeiros em fases distintas do seu desenvolvimento, completando assim os seus ciclos de vida (Blaber, 2002). Por exemplo, várias espécies da família Sciaenidae utilizam habitats estuarinos quando 
juvenis e áreas marinhas adjacentes quando adultas (CHAO \& Musick, 1977; Снао et al. 1982). Espécies recifais geralmente apresentam uma intima conexão com habitats caracterizados por cobertura de algas e presença de gramíneas marinhas, localizados próximos aos recifes (NAGELKERKEN et al., 2000).

Essa conectividade de habitats tem sido considerada como um aspecto chave na concepção de redes integradas de áreas marinhas protegidas para a conservação da biodiversidade (MURRAY et al., 1999; FRIEDLANDER et al., 2003). Embora muitos estudos tenham se concentrado na identificação de habitats essenciais para a manutenção do ciclo de vida das espécies (STONER, 2006), ainda se conhece pouco sobre a importância e a conectividade entre habitats costeiros, seja esta com estuários adjacentes (ABLE, 2005) ou com outros habitats próximos, como a zona de arrebentação de praias arenosas e ambientes recifais.

A região costeira de Itaipu (Niterói), localizada junto à entrada da Baía de Guanabara (Fig. 1), é uma área peculiar na costa do Estado do Rio de Janeiro por concentrar diferentes habitats e receber continuamente contribuições de águas oceânicas e continentais (Salvador \& Silva, 2002). Esta área compreende um conjunto de ilhas costeiras que protege parcialmente uma enseada do batimento das ondas, influenciando a sedimentação e a morfologia do fundo (SALVADOR \& SiLVA, 2002) favorecendo a prática da pesca artesanal (KANT DE Lima \& PEREIRA, 1997). Essa pescaria, praticada por cerca de 260 pescadores, é responsável pela captura diária de uma grande variedade de espécies de peixes que procuram águas rasas e abrigadas. Apesar da importância desta atividade como fonte de emprego e renda (KANT DE Lima \& Pereira, 1997; Pessanha, 2003; Barbosa \& Begossi, 2004), poucos estudos têm focalizado as características da ictiofauna, bem como o uso destes recursos pela pesca.

Os recifes rochosos associados às ilhas da Menina, Mãe e Pai, são também habitats importantes na agregação de biodiversidade e biomassa local, incluindo a biota bentônica e peixes recifais, conforme exposto por FLOETER et al. (2007) para o Sudeste do Brasil. Muitos estudos com peixes recifais foram realizados em Arraial do Cabo, RJ (e. g. FerReira et al., 2001), porém pouca atenção tem sido dada a ictiofauna dos sistemas insulares adjacentes à Baia de Guanabara.

O complexo lagunar Itaipu-Piratininga, conectado constantemente à enseada por um canal, representa um habitat específico, propício à reprodução e/ou crescimento de espécies marinhas ou estuarino dependentes (sensu ABLE, 2005), muitas delas de interesse econômico (Sergipense \& Pinto, 1995).

Portanto, a região costeira de Itaipu constitui uma importante área de agregação, produção e constante troca de recursos biológicos. Reconhecer as conectividades locais possibilita a adoção de uma abordagem ecossistêmica na definição dos padrões de uso de habitats pelas espécies, constituindo uma valiosa informação para a definição de áreas protegidas e zoneamento costeiro (STONER, 2006). Este estudo apresenta uma análise exploratória das associações de peixes na região costeira de Itaipu, identificando as relações de conectividade entre a atividade pesqueira praticada na enseada e os demais habitats (zona de arrebentação de praia, ilhas costeiras e lagoa de Itaipu).

\section{MATERIAL E MÉTODOS}

A região costeira de Itaipu está localizada na orla oceânica do município de Niterói, Rio de Janeiro ( $22^{\circ} 53^{\prime} 14^{\prime \prime}$ 'S, $\left.43^{\circ} 22^{\prime} 48^{\prime \prime} \mathrm{W}\right)$. Sua linha de costa ocupa uma extensão de 4,5km e apresenta uma formação semicircular no sentido E-W, sendo dividida na sua porção mediana pelo canal da lagoa de Itaipu. O perfil da praia é, na sua maior parte, íngreme e reflexivo, com profundidades entre 3 a $28 \mathrm{~m}$ e uma cobertura sedimentar homogênea, constituída predominantemente por areia média com alguns pontos de depósitos arenosos mais finos provenientes do canal de Itaipu (SALVAdOR \& SiLVA, 2002). A enseada é limitada em direção ao oceano pelo alinhamento das ilhas do Pai, da Mãe e da Menina (Fig. 1).

As informações apresentadas são provenientes dos seguintes programas de monitoramento da ictiofauna conduzidos na localidade:

I. Pesca (Projeto ITAPESQ-MCT-PADCT). Acompanhamento quinzenal dos desembarques da pesca artesanal local entre abril de 2001 e março de 2003. Foram identificadas as espécies desembarcadas e anotados procedência e petrechos de pesca utilizados. São consideradas neste estudo as pescarias realizadas com arrasto-de-praia, rede de emalhe e linha e anzol.

II. Zona de arrebentação. Coletas mensais com arrastos-de-praia em três pontos na zona de arrebentação da praia de Itaipu (Fig. 1), entre julho de 2002 e março de 2003 , totalizando 36 amostras. Os arrastos, conduzidos paralelamente à linha de costa, foram realizados com rede do tipo picaré com $16 \mathrm{~m}$ de comprimento, $2,5 \mathrm{~m}$ de altura e malha de $10 \mathrm{~mm}$ entre nós adjacentes.

III. Ilhas costeiras. Censos visuais sub-aquáticos mensais realizados nas ilhas da Menina, da Mãe e do Pai, entre março de 2002 e abril de 2003, totalizando 36 perfis amostrais. O método de amostragem seguiu os procedimentos descritos por FLOETER et al. (2001), modificando-se o comprimento do perfil para $40 \mathrm{~m}$.

IV. Lagoa de Itaipu. Amostras obtidas com a utilização dos seguintes petrechos de pesca: (a) três redes

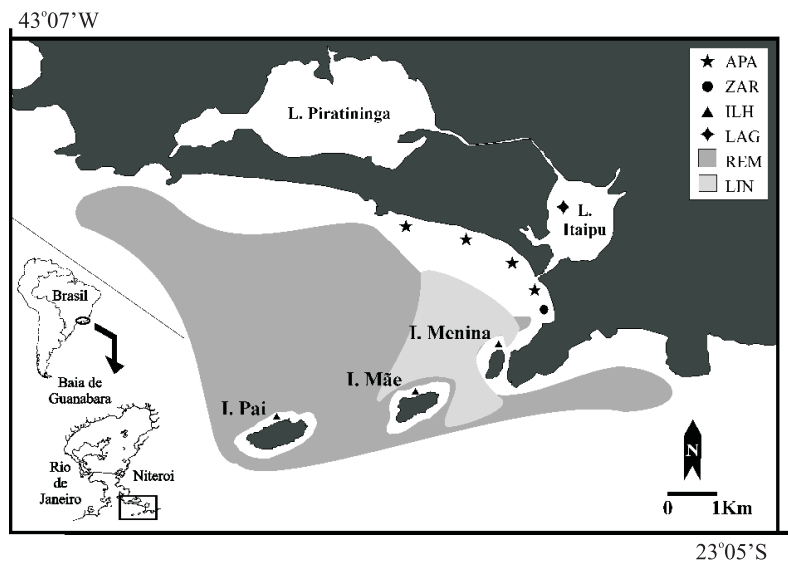

Fig. 1. Localização das áreas de coleta de dados na região costeira de Itaipu, Niterói, RJ (APA, arrasto-de-praia artesanal; REM, rede de emalhe; LIN, linha de mão; ZAR, zona de arrebentação; ILH, ilhas costeiras (Menina, Mãe e Pai) e LAG, lagoa de Itaipu). 
de emalhe (50m x $2 \mathrm{~m}$ cada), com malhas de $12 \mathrm{~mm}, 20 \mathrm{~mm}$ e $35 \mathrm{~mm}$ entre nós adjacentes respectivamente; (b) tarrafas de $32,1 \mathrm{~m}^{2}$ de área e malhas de $12 \mathrm{~mm}$ e $20 \mathrm{~mm}$ entre nós adjacentes; (c) armadilhas confeccionadas com garrafas PET 21 e (d) puçá com malha de $1 \mathrm{~mm}$ entre nós adjacentes.

A identificação dos exemplares baseou-se na bibliografia para peixes marinhos da costa atlântica sulamericana (FIgUeIREdo, 1977; FigueIREdo \& MENEZES, 1978, 1980, 2000; Menezes \& Figueiredo, 1980, 1985; Carvalho-Filho, 1999; Humann \& Deloach, 2002). Os nomes científicos e populares foram padronizados de acordo com a base Fishbase (http://www.fishbase.org, acesso em 08/08/2007).

Os registros de ocorrência das espécies foram agrupados em uma matriz de presença/ausência, considerando suas presenças nos diferentes sistemas de capturas da pesca artesanal (APA - arrasto-de-praia; REM - redes de emalhe corvineira, linguadeira e alta, descritas por KANT DE LIMA \& PEREIRA (1997); LIN - linha de fundo) e habitats (ZAR - zona de arrebentação; ILH ambiente recifal das ilhas costeiras; LAG - lagoa de Itaipu).

A partir de uma matriz contendo apenas espécies que co-ocorreram em mais de um sistema de captura/ habitat, aplicou-se uma análise de agrupamento em modo $\mathrm{Q}$, para identificar as relações de similaridade entre os mesmos. Para estabelecer os padrões de associações hierárquicas entre grupos de espécies aplicou-se a mesma matriz de dados, procedimento similar no modo R. Em ambas as análises empregou-se o percentual de discordância como medida de semelhança entre os objetos (STATSOFT, 2006) e o algorítmo de Ward como critério de agrupamento na construção dos dendrogramas (Milligan \& CoOper, 1987).

Para melhor descrever essas relações, os resultados das análises de agrupamento (modos Q e R) foram correlacionados entre si através de uma tabela de contingência (análise nodal) utilizando-se os conceitos de constância e fidelidade ecológica (BoEsch, 1977). A constância é expressa pela frequiência de ocorrência de um grupo de espécies em um determinado grupo de amostras, enquanto que a fidelidade traduz o quanto um grupo de espécies é restrito a um determinado grupo de amostras, comparando-se a sua constância individual com a constância média obtida entre todos os grupos (BOESCH, 1977; Musick et al., 1985; MonTEIRO-Neto et al., 2003).

A técnica de análise nodal permite a otimização das associações intragrupos, através da realocação das espécies ou amostras entre os grupos, minimizando assim artifícios de classificação inerentes ao método de análise (BOESCH, 1977).

Para otimização gráfica, foram estabelecidas escalas de categorias para constância (alta: > 0,7; moderada: 0,5-0,7 e baixa: <0,5) e fidelidade ecológica (alta: > 2,0; moderada: 1,0-2,0 e baixa: < 1,0). Uma quarta categoria representa a ausência de um grupo de espécies em uma dimensão.

\section{RESULTADOS}

Foram registradas 183 espécies de peixes para a região costeira de Itaipu, sendo 26 Elasmobranchii (13 famílias e 20 gêneros) e 157 espécies de Actinopterygii (63 famílias e 113 gêneros) (Tab. I). A pesca artesanal de arrasto-de-praia (APA) registrou o maior número de espécies (112) sendo 10 exclusivas. As pescarias realizadas com os diferentes tipos de redes de emalhe, também apresentaram alta riqueza, totalizando 94 espécies (18 delas exclusivas desta arte) representadas principalmente por cações e raias. As pescarias de linha registraram apenas 35 espécies, sendo duas exclusivas (Conger orbignyanus Valenciennes, 1847 e Pteroplatytrygon violacea (Bonaparte, 1832)), raramente encontradas na região. Os arrastos com picaré na zona de arrebentação capturaram 49 espécies ( 8 exclusivas) e os censos visuais realizados nas ilhas identificaram um conjunto de 41 espécies, 21 de caráter exclusivo. Quarenta e seis espécies foram registradas para a lagoa de Itaipu, sendo que deste total, 18 apresentaram ocorrência exclusiva (Tab. I).

A análise de agrupamento no modo $\mathrm{Q}$ revelou a formação de três grupos distintos a um nível de discordância de $50 \%$. As principais artes de pesca praticadas na enseada (APA e REM) apresentaram a menor discordância entre si. Um segundo grupo foi formado pelas pescarias de linha de mão e os censos visuais efetuados nas ilhas costeiras. O terceiro grupo foi composto pela zona de arrebentação e a lagoa de Itaipu (Fig. 2). A classificação em modo R revelou inicialmente 12 grupos de espécies, que submetidos à análise nodal, foram consolidados em oito, incluindo um total de 106 espécies (Tab. I, grupos A-H). As espécies exclusivas (77) foram reunidas de acordo com as suas ocorrências nas respectivas dimensões amostrais (Tab. I, grupos I-N).

O grupo A foi constituído por 23 espécies com ocorrências restritas ao arrasto-de-praia (APA) e as redes de emalhe (REM), resultando em valores altos de constância e fidelidade (Fig. 3). Estão incluídos nesse grupo os elasmobrânquios Dasyatis say (Lesurur, 1817), Mobula hypostoma (Bancroft, 1831), Gymnura altavela (Linnaeus, 1758), Mustelus schmitti Springer, 1939, Narcine brasiliensis (Olfers, 1831), Sphyrna lewini (Griffith \& Smith, 1834), Squatina guggenheim Marini, 1936, S. occulta Vooren \& da Silva, 1992 e actinopterígios das famílias Sciaenidae (Larimus breviceps (Cuvier, 1830), Paralonchurus brasiliensis (Steindachner, 1875), Umbrina canosai Berg, 1895), Scombridae (Sarda sarda (Bloch, 1793), Scomberomorus cavalla (Cuvier, 1829), S. brasiliensis Collette, Russo \& Zavala-Camin, 1978) e

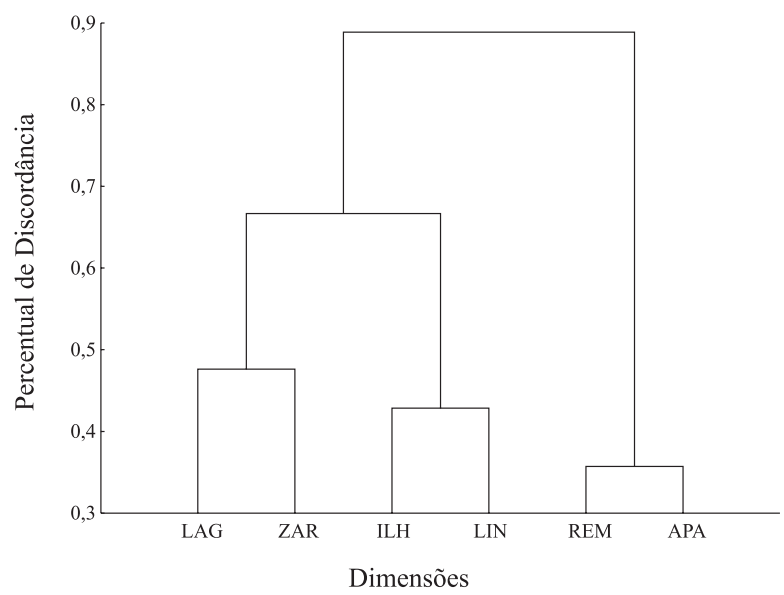

Fig. 2. Dendrograma da análise de agrupamento dos sistemas de captura e habitats monitorados na região costeira de Itaipu, Niterói, RJ (APA, arrasto-de-praia; REM, rede de emalhe; LIN, linha de mão; ZAR, zona de arrebentação; ILH, ilhas costeiras e LAG, lagoa de Itaipu). 
Tabela I. Grupos de espécies e ocorrência nos sistemas de captura e habitats monitorados na região costeira de Itaipu, Niterói, RJ (APA, arrasto-de-praia; REM, rede de emalhe; LIN, linha de mão; ZAR, zona de arrebentação; ILH, ilhas costeiras e LAG, lagoa de Itaipu). Grupos A-H são provenientes da análise nodal e I-N são as espécies exclusivas reunidas de acordo com as suas ocorrências nas respectivas dimensões amostrais.

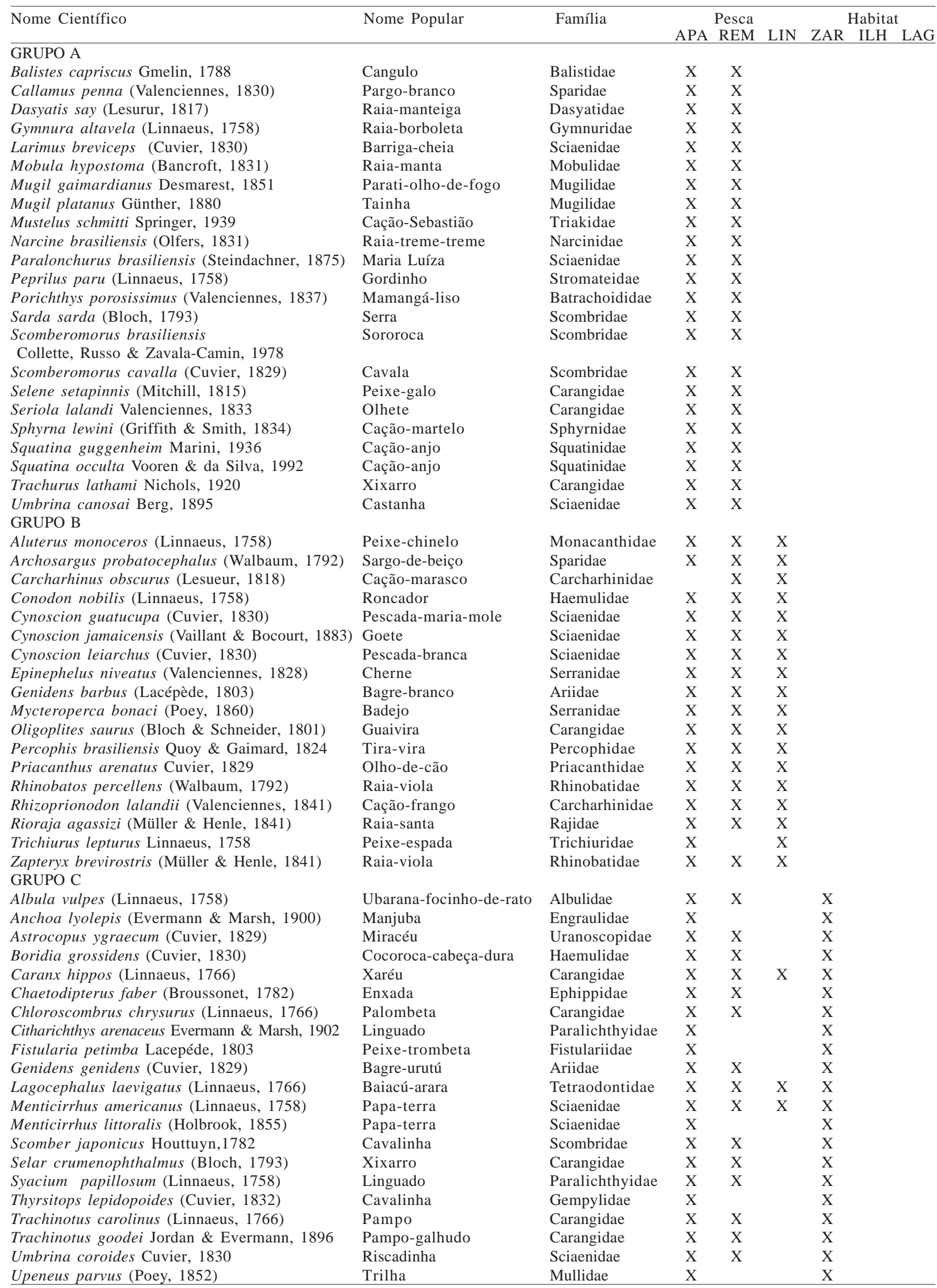


Tabela I (cont.)

\section{GRUPO D}

Anisotremus virginicus (Linnaeus, 1758)

Caranx crysos (Mitchill, 1815)

Dactylopterus volitans (Linnaeus, 1758)

Epinephelus marginatus (Lowe, 1834)

Gymnothorax ocellatus Agassiz, 1831

Haemulon steindachneri (Jordan \& Gilbert, 1882)

Hippocampus erectus Perry, 1810

Lutjanus analis (Cuvier, 1828)

Pagrus pagrus (Linnaeus, 1758)

Pseudupeneus maculatus (Bloch, 1797)

GRUPO E

Archosargus rhomboidalis (Linnaeus, 1758)

Caranx latus Agassiz, 1829

Diapterus auratus Ranzani, 1842

Diapterus rhombeus (Cuvier, 1829)

Diplectrum formosum (Linnaeus, 1766)

Diplectrum radiale (Quoy \& Gaimard, 1824)

Elops saurus Linnaeus, 1766

Micropogonias furnieri (Desmarest, 1823)

Mugil curema Valenciennes, 1836

Mugil liza Valenciennes, 1836

Opisthonema oglinum (Lesueur, 1818)

Sphoeroides testudineus (Linneaus, 1758)

GRUPO F

Achirus lineatus (Linnaeus, 1758)

Anchoviella lepidentostole (Fowler, 1911)

Atherinella brasiliensis (Chernoff, 1986)

Centropomus undecimalis (Bloch, 1792)

Cetengraulis edentulus (Cuvier, 1828)

Eucinostomus argenteus Baird \& Girard, 1855

Harengula clupeola (Cuvier, 1829)

Pomatomus saltatrix (Linneaus, 1766)

Prionotus punctatus (Bloch, 1797)

Sardinella janeiro (Eigenmann, 1894)

Selene vomer (Linneaus, 1758)

Sphyraena tome Fowler, 1903

GRUPO G

Bothus ocellatus (Agassiz, 1831)

Diplodus argenteus (Valeciennes, 1830)

Orthopristis ruber (Cuvier, 1830)

Scorpaena isthmensis Meek \& Hildebrand, 1928

Sphoeroides spengleri (Bloch, 1785)

Stephanolepis hispidus (Linnaeus, 1758)

GRUPO H

Chilomycterus spinosus (Linnaeus, 1758)

Eucinostomus gula (Quoy \& Gaimard, 1824)

Sphoeroides greeleyi Gilbert, 1900

Synodus foetens (Linnaeus, 1766)

ESPÉCIES EXCLUSIVAS

GRUPO I

Ctenosciaena gracilicirrhus (Metezlaar, 1919)

Salema

Xerelete

Coió

Garoupa

Moreia-pintada

Cocoroca

Cavalo-marinho

Vermelho-caranho

Pargo-rosa

Trilha

Canhanha

Faqueco

Carapeba

Carapeba

Michole-de-areia

Michole-de-areia

Ubarana

Corvina

Parati

Tainha

Sardinha-laje

Baiacú

Linguado

Manjuba

Peixe-rei

Robalo

Sardinha-boca-torta

Carapicu

Sardinha-cascuda

Enchova

Cabrinha

Sardinha-verdadeira

Galo-de-penacho

Bicuda

Linguado

Marimbá

Cocoroca

Peixe-pedra

Baiacú

Peixe-porco

Baiacu-espinho

Carapicu

Baiacu-pinima

Peixe-lagarto

Barriga-cheia

Pescada-cabuçu

Chicharro-cobra

Rêmora

Bonito-pintado

Peixe-trombeta

Linguado

Cocoroca-boca-de-fogo

Sardinha da noite

Bicuda

Raia-pintada

Raia-fumo

Cação-galha-preta

Raia

Raia-sapo

Aniquim

Bonito-listrado

Pirangica

$\begin{array}{llccc}\text { Haemulidae } & & \text { X } & & \text { X } \\ \text { Carangidae } & \text { X } & \text { X } & \text { X } & \text { X } \\ \text { Dactylopteridae } & \text { X } & & & \text { X } \\ \text { Serranidae } & \text { X } & \text { X } & \text { X } & \text { X } \\ \text { Muraenidae } & \text { X } & & & \text { X } \\ \text { Haemulidae } & \text { X } & & & \text { X } \\ \text { Syngnathidae } & \text { X } & & & \text { X } \\ \text { Lutjanidae } & \text { X } & \text { X } & \text { X } & \text { X } \\ \text { Sparidae } & & \text { X } & & \text { X } \\ \text { Mullidae } & \text { X } & & & \text { X }\end{array}$

Sparidae

Carangidae

Gerreidae

Gerreidae

Serranidae

Serranidae

Elopidae

Sciaenidae

Mugilidae

Mugilidae

Clupeidae

Tetraodontidae

Achiridae

Engraulidae

Atherinopsidae

Centropomidae

Engraulidae

Gerreidae

Clupeidae

Pomatomidae

Triglidae

Clupeidae

Carangidae

Sphyraenidae

Bothidae

Sparidae

Haemulidae

Scorpaenidae

Tetraodontidae

Monacanthidae

Diodontidae

Gerreidae

Tetraodontidae

Synodontidae

$\begin{array}{llll}X & X & X\end{array}$

$\mathrm{X} \quad \mathrm{X}-\mathrm{X}-\mathrm{X}$

$\begin{array}{lll}X & X & X\end{array}$

$\begin{array}{llll}X & X & X \\ X & X & X & X \\ X & & X & X\end{array}$

$\mathrm{X}-\mathrm{X}$

$\mathrm{X} \quad \mathrm{X} \quad \mathrm{X}$

$\begin{array}{llll}X & X & X & x \\ X & x & x\end{array}$

$\mathrm{X} \quad \mathrm{X} \quad \mathrm{X}$

$\mathrm{X}-\mathrm{X}-\mathrm{X}$

$\mathrm{X}$

$\mathrm{X}$

$\mathrm{X}$

$\mathrm{X}$

$\mathrm{X}$

$\mathrm{X}$

$\mathrm{X}$

$\mathrm{X}$

$\mathrm{X}$

$\mathrm{X}$

$\mathrm{X}$

$\mathrm{X}$

$\mathrm{X}$

$\mathrm{X}$

$\mathrm{X}$
$\mathrm{X}$

Carangidae

Sciaenidae

Carangidae

Echeneididae

Scombridae

Fistulariidae

Achiridae

Haemulidae

Pristigasteridae $\mathrm{X}$

Sphyraenidae $\quad \mathrm{X}$

Myliobatidae

Rajidae

$\mathrm{X}$

Carcharhinidae

Gymnuridae

Myliobatidae

Lamnidae

Scombridae

Kiphosidae

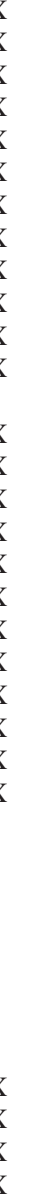

Kyphosus sectator (Linnaeus, 1758) 
Tabela I (cont.)

\begin{tabular}{|c|c|c|c|c|c|c|c|c|}
\hline \multirow[t]{2}{*}{ Nome Científico } & \multirow[t]{2}{*}{ Nome Popular } & \multirow[t]{2}{*}{ Família } & \multicolumn{3}{|c|}{ Pesca } & \multicolumn{3}{|c|}{ Habitat } \\
\hline & & & APA & REM & LIN & ZAR & ILH & LAG \\
\hline Lophius gastrophysus Miranda-Ribeiro, 1915 & Tamboril & Lophiidae & & $\mathrm{X}$ & & & & \\
\hline Rhinoptera bonasus (Mitchill, 1815) & Raia-ticonha & Myliobatidae & & $\mathrm{X}$ & & & & \\
\hline Rhizoprionodon porosus (Poey, 1861) & Cação-frango & Carcharhinidae & & $\mathrm{X}$ & & & & \\
\hline Sphyrna zygaena (Linnaeus, 1758) & Cação-martelo & Sphyrnidae & & $\mathrm{X}$ & & & & \\
\hline Squalus cf.cubenseis Howell Rivero, 1936 & Cação-bagre & Squalidae & & $\mathrm{X}$ & & & & \\
\hline Squatina dumeril Lesueur, 1818 & Cação-anjo & Squatinidae & & $\mathrm{X}$ & & & & \\
\hline Stellifer rastrier (Jordan, 1889) & Cangoa & Sciaenidae & & $\mathrm{X}$ & & & & \\
\hline Sympterygia acuta Garman, 1877 & Raia-santa & Rajidae & & $\mathrm{X}$ & & & & \\
\hline Urophycis brasiliensis (Kaup, 1858) & Abrótea & Gadidae & & $\mathrm{X}$ & & & & \\
\hline $\begin{array}{l}\text { Zenopsis conchifer (Lowe, 1850) } \\
\text { GRUPO K }\end{array}$ & Galo-cara-de-cavalo & Zeidae & & $\mathrm{X}$ & & & & \\
\hline Conger orbignyanus Valenciennes, 1847 & Congro & Congridae & & & $\mathrm{X}$ & & & \\
\hline $\begin{array}{l}\text { Pteroplatytrygon violacea (Bonaparte, 1832) } \\
\text { GRUPO L }\end{array}$ & Raia-mero & Dasyatidae & & & $\mathrm{X}$ & & & \\
\hline Bothus robinsi Topp \& Hoof, 1972 & Linguado & Bothidae & & & & $\mathrm{X}$ & & \\
\hline Etropus crossotus Jordan \& Gilbert, 1882 & Linguado & Paralichthyidae & & & & $\mathrm{X}$ & & \\
\hline Polydactylus oligodon (Günther, 1860) & Parati-barbudo & Polynemidae & & & & $\mathrm{X}$ & & \\
\hline Polydactylus virginicus (Linnaeus, 1758) & Parati-barbudo & Polynemidae & & & & $\mathrm{X}$ & & \\
\hline Synodus intermedius (Spix \& Agassiz, 1829) & Peixe-lagarto & Synodontidae & & & & $\mathrm{X}$ & & \\
\hline Trachinotus falcatus (Linnaeus, 1758) & Pampo & Carangidae & & & & $\mathrm{X}$ & & \\
\hline Trachinotus marginatus Cuvier, 1832 & Pampo & Carangidae & & & & $\mathrm{X}$ & & \\
\hline Trachynocephalus myops (Forster, 1801) & Peixe-lagarto & Synodontidae & & & & $X$ & & \\
\hline GRUPO M & & & & & & & & \\
\hline Abudefduf saxatilis (Linnaeus, 1758) & Sargento & Pomacentridae & & & & & $\mathrm{X}$ & \\
\hline Acanthurus chirurgus (Bloch, 1787) & Cirurgião & Acanthuridae & & & & & $\mathrm{X}$ & \\
\hline Chaetodon sedentarius Poey, 1860 & Peixe-borboleta & Chaetodontidae & & & & & $\mathrm{X}$ & \\
\hline Chaetodon striatus Linnaeus, 1758 & Peixe-borboleta & Chaetodontidae & & & & & $\mathrm{X}$ & \\
\hline Chromis multilineata (Guichenot, 1855) & Donzela-de-recife & Pomacentridae & & & & & $\mathrm{X}$ & \\
\hline Diodon hystrix Linnaeus, 1758 & Baiacu & Diodontidae & & & & & $\mathrm{X}$ & \\
\hline Halichoeres cyanocephalus (Bloch, 1791) & Sabonete & Labridae & & & & & $\mathrm{X}$ & \\
\hline Halichoeres poeyi (Steindachner, 1867) & Sabonete & Labridae & & & & & $\mathrm{X}$ & \\
\hline Heteropriacanthus cruentatus (Lacepède, 1801) & Olho-de-cão & Priacanthidae & & & & & $\mathrm{X}$ & \\
\hline Hipросатриs reidi Ginsburg, 1933 & Cavalo-marinho & Syngnathidae & & & & & $\mathrm{X}$ & \\
\hline Labrisomиs nисhipinnis (Quoy \& Gaimard, 1824) & Maria-da-toca & Labrisomidae & & & & & $\mathrm{X}$ & \\
\hline Mycteroperca acutirostris (Poey, 1861) & Badejo-quadrado & Serranidae & & & & & $\mathrm{X}$ & \\
\hline Myripristis jacobus Cuvier, 1839 & Vernelho & Holocentridae & & & & & $\mathrm{X}$ & \\
\hline Parablennius marmoreus (Рoey, 1876) & Maria-da-toca & Bleniidae & & & & & $\mathrm{X}$ & \\
\hline Parablennius pilicornis (Cuvier, 1829) & Maria-da-toca & Bleniidae & & & & & $\mathrm{X}$ & \\
\hline Pareques acuminatus (Bloch \& Schneider, 1801) & Anteninha & Sciaenidae & & & & & $\mathrm{X}$ & \\
\hline Pomacanthus paru (Bloch, 1787) & Peixe-frade & Pomacanthidae & & & & & $\mathrm{X}$ & \\
\hline Pseudocaranx dentex (Bloch \& Schneider, 1801) & Xaréu & Carangidae & & & & & $\mathrm{X}$ & \\
\hline Scartella cristata (Linnaeus, 1758) & Maria-da-toca & Bleniidae & & & & & $\mathrm{X}$ & \\
\hline Sparisoma axillare (Steindachner, 1878) & Budião & Scaridae & & & & & $\mathrm{X}$ & \\
\hline Stegastes fuscus (Cuvier, 1830) & Donzela & Pomacentridae & & & & & $\mathrm{X}$ & \\
\hline GRUPO N & & & & & & & & \\
\hline Anchoa tricolor (Spix \& Agassiz, 1829) & Manjuba & Engraulidae & & & & & & $\mathrm{X}$ \\
\hline Anchovia clupeoides (Swainson, 1939) & Manjuba & Engraulidae & & & & & & $\mathrm{X}$ \\
\hline Bathygobius soporator (Valeciennes, 1837) & Maria-da-toca & Gobiidae & & & & & & $\mathrm{X}$ \\
\hline Brevoortia aurea (Spix \& Agassiz, 1829) & Savelha & Clupeidae & & & & & & $\mathrm{X}$ \\
\hline Centropoтиs parallelus Poey, 1860 & Robalo & Centropomidae & & & & & & $\mathrm{X}$ \\
\hline Citharichthys spilopterus Günther, 1862 & Linguado & Paralichthyidae & & & & & & $\mathrm{X}$ \\
\hline Cynoscion acoupa (Lacepède, 1801) & Pescada & Sciaenidae & & & & & & $\mathrm{X}$ \\
\hline Eucinostomus lefroyi (Goode, 1874) & Carapicu & Gerreidae & & & & & & $\mathrm{X}$ \\
\hline Eucinostomus melanopterus (Bleeker, 1863) & Carapicu & Gerreidae & & & & & & $\mathrm{X}$ \\
\hline Gobionellus oceanicus (Pallas, 1770) & Maria-da-toca & Gobiidae & & & & & & $\mathrm{X}$ \\
\hline Gobionellus shufeldti (Jordan \& Eigenmann, 1887) & Maria-da-toca & Gobiidae & & & & & & $\mathrm{X}$ \\
\hline Gobionellus stomatus Starks, 1913 & Maria-da-toca & Gobiidae & & & & & & $\mathrm{X}$ \\
\hline Haemulon plumierii (Lacepède, 1801) & Cocoroca & Haemulidae & & & & & & $\mathrm{X}$ \\
\hline Jenynsia multidentata (Jenyns, 1842) & Barrigudinho & Anablepidae & & & & & & $\mathrm{X}$ \\
\hline Oreochromis niloticus (Linnaeus, 1758) & Tilápia-do-Nilo & Cichlidae & & & & & & $\mathrm{X}$ \\
\hline Poecilia vivipara Bloch \& Schneider, 1801 & Barrigudinho & Poeciliidae & & & & & & $\mathrm{X}$ \\
\hline Stephanolepis setifer (Bennett, 1831) & Peixe-porco & Monacanthidae & & & & & & $\mathrm{X}$ \\
\hline Symphurus plagusia (Bloch \& Schneider, 1801) & Língua-de-mulata & Cynoglossidae & & & & & & $\mathrm{X}$ \\
\hline
\end{tabular}

Carangidae (Selene setapinnis (Mitchill, 1815), Seriola lalandi Valenciennes, 1833, Trachurus lathami Nichols, 1920), entre outras (Tab. I).

O grupo B, composto por 18 espécies capturadas nas três artes de pesca (APA, REM e LIN) apresentou constância alta e fidelidade moderada em APA e REM, porém alta em LIN (Fig. 3). Os elasmobrânquios (e. $g$. Carcharinus obscurus (Lesueur, 1818), Rhinobatos percellens (Walbaum, 1792), Rhizoprionodon lalandii (Valenciennes, 1841), Rioraja agassizi (Müller \& Henle, 
1841), Zapteryx brevirostris (Müller \& Henle, 1841)) e os actinopterígios da família Sciaenidae, em especial as pescadas (Cynoscion spp.), Serranidae (Epinephelus niveatus (Valenciennes, 1828) e Mycteroperca bonaci (Poey, 1860)), bem como a espada (Trichiurus lepturus Linnaeus, 1758, Trichiuridae), foram as espécies mais representativas do grupo (Tab. I).

De uma forma geral, os grupos A e B se caracterizam por espécies de hábitos pelágicos e demersais que ocorrem na enseada mas não apresentam forte conexão com os outros habitats costeiros monitorados.

O grupo C apresentou constância alta e fidelidade moderada no arrasto de praia, na rede de emalhe e na zona de arrebentação (ZAR), porém ambos os índices foram baixos para a linha de mão (LIN) (Fig. 3). Neste grupo estão representadas 21 espécies, na sua maioria ocorrendo como juvenis na zona de arrebentação e cujos jovens e adultos participam das capturas em pescarias locais. As famílias Carangidae (5 espécies) e Sciaenidae (3 espécies), foram as mais representativas. Menticirrhus littoralis (Holbrook, 1855), M. americanus (Linnaeus, 1758), Chloroscombrus chrysurus (Linnaeus, 1766), Trachinotus carolinus (Linnaeus, 1766), T. goodei (Jordan \& Evermann, 1896) e o bagre Genidens genidens (Cuvier, 1829, Ariidae) fazem parte deste grupo (Tab. I).

O grupo D incluiu nove espécies cujas ocorrências se restringiram à pesca artesanal e às ilhas, apresentando constância alta nesse habitat e no arrasto de praia (APA). A fidelidade alta só foi verificada em ILH (Fig. 3). As espécies contidas neste grupo são típicas de ambientes recifais (Epinephelus marginatus (Lowe, 1834), Gymnothorax ocellatus Agassiz, 1831, Haemulon steindachneri (Jordan \& Gilbert, 1882), Hippocampus erectus Perry, 1810) e/ou de ambientes arenosos próximos (Pseudupeneus maculatus (Bloch, 1797), Dactylopterus volitans (Linnaeus, 1758)) (Tab. I).

$\mathrm{O}$ grupo $\mathrm{E}$ agregou 12 espécies comuns à pesca artesanal e à lagoa de Itaipu, incluindo os mugilídeos (Mugil liza Valenciennes, 1836, M. curema Valenciennes, 1836), a corvina (Micropogonias furnieri (Desmarest, 1823)) e as carapebas (Diapterus rhombeus (Cuvier, 1829) e D. auratus Ranzani, 1842) (Tab. I). As espécies contidas neste grupo apresentam constância alta na lagoa e nas pescas com arrasto-de-praia e rede de emalhe (Fig. 3).

$\mathrm{O}$ grupo $\mathrm{F}$ foi formado por 12 espécies com ocorrência na pesca (APA, REM e LIN), na zona de arrebentação (ZAR) e na lagoa de Itaipu (LAG). A constância deste grupo apresentou-se alta nas dimensões APA, ZAR e LAG; porém a fidelidade variou de moderada a baixa (Fig. 3). Este grupo foi composto por espécies predominantemente pelágicas de pequeno porte, como Anchoviella lepidentostole (Fowler, 1911), Sardinella janeiro (Eigenmann, 1894), Cetengraulis edentulus (Cuvier, 1828) e Atherinella brasiliensis (Chernoff, 1986) (Tab. I); peixes característicos de ambientes lagunares, estuarinos e de regiões costeiras. Outras espécies como Pomatomus saltatrix (Linneaus, 1766) e Eucinostomus argenteus Baird \& Girard, 1855, são encontradas como juvenis nesses ambientes e participam - como jovens ou adultos - nas pescarias locais.

O grupo $G$ foi constituído por 6 espécies e apresentou constância alta e fidelidade moderada em APA, ZAR e ILH, não ocorrendo na lagoa. Fizeram parte deste grupo Diplodus argenteus (Valeciennes, 1830), Orthopristis ruber (Cuvier, 1830), Stephanolepis hispidus (Linnaeus, 1758) e Sphoeroides spengleri (Bloch, 1785).

$\mathrm{O}$ menor grupo formado foi o grupo $\mathrm{H}$, constituído apenas por 4 espécies com ampla distribuição espacial, ocorrendo na pesca, na zona de arrebentação, nas ilhas e na lagoa. Apesar da alta constância em APA, ILH e LAG, este grupo apresentou fidelidade apenas moderada e baixa em todas as dimensões (Fig. 3). Estão incluídas neste grupo Chilomycterus spinosus (Linnaeus, 1758), Eucinostomus gula (Quoy \& Gaimard, 1824), Synodus foetens (Linnaeus, 1766) e Sphoeroides greeleyi Gilbert, 1900 (Tab. I).

As artes de pesca que atuam na enseada (APA, REM, LIN) acumularam 30 espécies exclusivas (Tab. I, grupos I-K), sendo que as redes de emalhe registraram mais da metade deste total. Na zona de arrebentação
A) CONSTÂNCIA

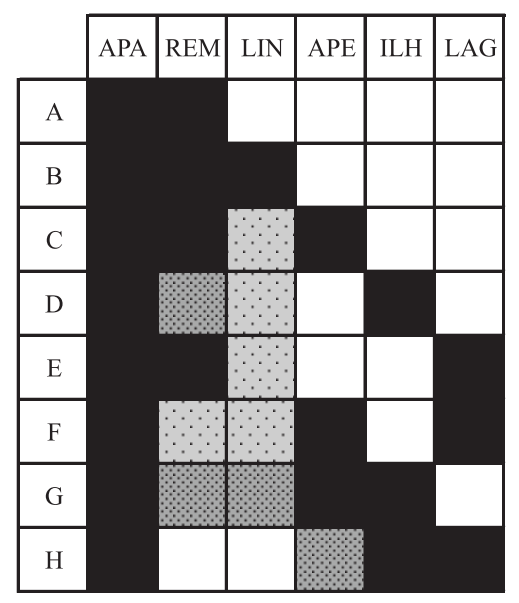

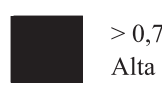

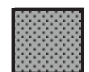

$0,5-0,7$ Moderada

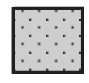

$<0,5$ Baixa

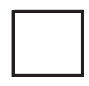

Ausente
B) FIDELIDADE

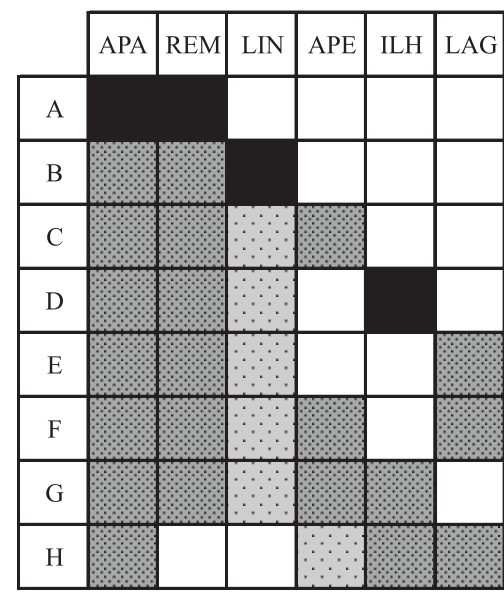

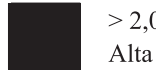

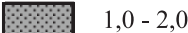

Moderada
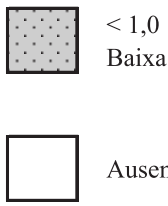

Ausente

Fig. 3. Padrões nodais de constância (A) e fidelidade ecológica (B) entre os grupos de espécies (A-H) e sistemas de captura (APA, arrastode-praia; REM, rede de emalhe; LIN, linha de mão) e habitat (ZAR, zona de arrebentação; ILH, ilhas costeiras; LAG, lagoa de Itaipu) monitorados na região costeira de Itaipu, Niterói, RJ. 
registrou-se apenas 8 espécies exclusivas, enquanto que em ILH e LAG observou-se um número mais elevado (Tab. I, grupos L-N).

\section{DISCUSSÃO}

A região costeira de Itaipu apresenta alta riqueza de espécies, acompanhando a tendência descrita por VIEIRA \& Musick (1993) para ambientes estuarinos tropicais. O elevado número de espécies registrado em Itaipu pode ser explicado em parte pela sua localização junto ao limite norte da Província Zoogeográfica Argentina, considerada como uma região de transição entre a fauna da zona temperada ao sul e da zona tropical ao norte (BRIGGS, 1974; FlOETER et al. 2001).

O uso de estratégias amostrais tão diversificadas possivelmente contribuiu para que a diversidade de espécies em Itaipu fosse elevada em relação a outras áreas costeiras. Araúso \& Azevedo (2001) observaram que comparações quanto à diversidade da ictiofauna entre diferentes estudos nem sempre é possível devido às diferenças entre habitats, programas amostrais e esforços de coleta. No entanto, trabalhos que avaliaram a ictiofauna em áreas costeiras no sudeste do Brasil, utilizando diferentes métodos de amostragem, apresentaram riqueza de espécies muito próximas dos valores obtidos em Itaipu. ANDREATA et al. (2002) registraram 148 espécies na Baía da Ribeira (RJ), enquanto que PAIVA-FILHO et al. (1987), amostrando o complexo baía-estuário de Santos, forneceram uma lista de 140 espécies de peixes.

As similaridades entre as diferentes dimensões monitoradas (APA, REM, LIN, ZAR, ILH e LAG) sugerem a existência de um padrão de conectividade entre elas baseado em fatores como proximidade e função ecológica dos habitats. Padrões de distribuição das espécies e seleção de habitats foram observados em comunidades de peixes recifais onde diferentes espécies utilizam áreas lagunares não estuarinas e áreas recifais, atendendo aos seus requerimentos ecológicos em diferentes tempos do ciclo de vida (Gratwicke et al., 2006). Able (2005) observou que diferentes habitats ao longo de um gradiente estuário-zona costeira, têm importante significado funcional para as espécies que coexistem ao longo desse gradiente. Em Itaipu, a pesca artesanal (principalmente APA e REM) amostrou especificamente o ambiente costeiro de enseada, caracterizado por um fundo predominantemente arenoso (SALVADOR \& Silva, 2002) e profundidades variando entre a linha de praia até cerca de $25 \mathrm{~m}$. O grande número de espécies registradas (aproximadamente $81 \%$ do total) sugere que a enseada funcione como uma ampla área de conexão entre os demais habitats costeiros monitorados. Predominaram nessas capturas espécies demersais (e.g. Micropogonias furnieri) e pelágicas de passagem (e. g. Sardinella janeiro, Caranx crysos (Mitchill, 1815)). O hábitat que apresentou o maior percentual de compartilhamento de espécies com a enseada foi a zona de arrebentação $(22,4 \%)$, seguida pela lagoa $(15,3 \%)$ e pelas ilhas costeiras $(10,9 \%)$.

O arrasto-de-praia apresentou a menor seletividade, capturando $60,5 \%$ do total de espécies. Além das espécies alvo, esta modalidade atinge uma variedade de espécies acessórias de pequeno porte e baixo valor comercial.
Padrão semelhante foi observado por LAMBERTH et al. (1997) na África do Sul e por Gray \& KenNelly (2003) na Austrália.

Os arrastos com picaré na zona de arrebentação amostraram uma faixa do sublitoral até $1,2 \mathrm{~m}$ de profundidade, com batimento de ondas variando entre fraco a moderado. São característicos dessa região os juvenis de muitas espécies marinhas que utilizam esta área para criação e proteção (LAYMAN, 2000; METHVEN et al., 2001; MonTeiro-Neto et al., 2003; Felix et al., 2007). Apesar da ocorrência de espécies típicas da zona de arrebentação (Trachinotus spp., Menticirrhus littoralis), observou-se uma participação relevante dos Clupeiformes (Anchoviella lepidentostole, Harengula clupeola (Cuvier, 1829) e Cetengraulis edentulus), também encontrados na lagoa de Itaipu. Felix et al. (2007) propõem que zonas de arrebentação funcionam como um habitat acessório a ambientes estuarinos, com função de berçário às espécies transientes, como os Clupeiformes. A presença de um número reduzido de espécies exclusivas na zona de arrebentação indica uma ampla conectividade desta assembléia de peixes (tanto com a enseada onde a pesca é praticada como com os demais habitats).

Mais da metade das espécies que ocorreram no ambiente recifal das ilhas da Menina, Mãe e Pai foram exclusivas, demonstrando o caráter particular da ictiofauna recifal, incluindo-se aqui as espécies criptobênticas ( $e . g$. Blenniidae, Syngnathidae) ou de hábitos de vida associados a ambientes rochosos $(e . g$. Pomacentridae, Labrisomidae) (FERreira et al., 2001; RANGEL et al., 2005). As espécies que apresentaram maior conectividade com a enseada - conseqüentemente ocorrendo nas pescarias - geralmente habitam fundos de areia (Dactylopterus volitans) ou tem grande mobilidade (Diplodus argenteus, Orthopristis ruber), deslocando-se freqüentemente entre os recifes e outros habitats adjacentes (NAGELKERKEN et al., 2000; GRATWICKE et al., 2006).

Entre as espécies registradas exclusivamente na lagoa de Itaipu, Jenynsia multidentata (Jenyns, 1842) e Poecilia vivipara Bloch \& Schneider, 1801 são consideradas estuarino-residentes (MONTEIRO-NETO et al., 1990), enquanto que Eucinostomus spp., Centropomus parallelus (Poey, 1860) e os Clupeiformes (Anchoa tricolor (Spix \& Agassiz, 1829), Anchovia clupeoides (Swainson, 1939), Brevoortia aurea (Spix \& Agassiz, 1829)) são estuarino-dependentes (ABLE, 2005). A lagoa de Itaipu apresenta um canal de ligação permanente com a enseada, permitindo o trânsito de espécies e indivíduos. Isso explica o fato do grupo $\mathrm{E}$ (formado majoritariamente por epipelágicos com hábito estuarino-lagunar) apresentar alta constância, sugerindo uma forte conectividade entre a lagoa e a enseada. Tal conectividade pode ser observada pelo fato de $15,3 \%$ das espécies capturadas na enseada ocorrerem no interior da lagoa de Itaipu, que se apresenta como um ambiente mais protegido e de alta produtividade biológica, possibilitando a complementação do ciclo de vida das espécies (СНАO et al., 1982; ANDREATA et al., 1990). Espécies de interesse a pesca como os carapicus (Gerreidae) e as tainhas e paratis (Mugil liza e M. curema), possivelmente procuram a lagoa para desenvolvimento ou reprodução. Alterações de qualidade de água, associadas à diminuição da lâmina 
d'água no interior da lagoa podem ser apontados como fatores limitantes à sua utilização por um número maior de espécies (WASSERMANN \& AlvES, 2004).

O presente trabalho constitui a primeira contribuição para uma análise integrada da ictiofauna costeira em Itaipu. Apesar de BEGOSsI (2006) ter observado a resiliência dos pesqueiros locais por um período superior a 30 anos, o crescimento urbano acelerado dos municípios de Niterói e do Rio de Janeiro aparentemente ameaça a manutenção da diversidade da ictiofauna local, bem como a atividade de pesca artesanal. A maior contribuição de águas eutrofizadas decorrente da ocupação costeira desordenada tem levado à redução da qualidade ambiental de habitats como as lagoas de Piratininga e Itaipu (Wasserman \& Alves, 2004). Considerando a hipótese de conectividade dos habitats levantada no presente estudo, essa redução terá conseqüências importantes para as espécies de peixes que ocorrem na região. Assim, a adoção de medidas integradas de ordenamento da atividade pesqueira e conservação dos recursos naturais locais deve considerar os diferentes ambientes que compõem esta zona costeira, visando a manutenção das suas funcionalidades ecológicas.

Agradecimentos. Ao Ministério de Ciência e Tecnologia pelo suporte financeiro. À Coordenação de Aperfeiçoamento de Pessoal do Ensino Superior, ao Conselho Nacional de Desenvolvimento Científico e Tecnológico e à Fundação de Amparo a Pesquisa do Estado do Rio de Janeiro pela concessão de bolsas aos autores. Aos pescadores de Itaipu pela colaboração na obtenção de informações sobre a pesca, em especial o Sr. Aurelino Mattos e Souza (Mestre Cambuci). À Viviane B. Sant'Anna pelo auxílio na identificação de elasmobrânquios. Aos alunos do Bacharelado em Biologia Marinha da Universidade Federal Fluminense, pelo auxílio nas atividades de campo e laboratório.

\section{REFRÊNCIAS BIBLIOGRÁFICAS}

Able, K. W. 2005. A re-examination of fish estuarine dependence: Evidence for connectivity between estuarine and ocean habitats. Estuarine, Coastal and Shelf Science 64:5-17.

Andreata, J. V.; Barbiéri, L. R. R.; da Silva, M. H. C. \& dos Santos, R. O. 1990. Relação dos peixes da Laguna de Marapendi. Rio de Janeiro, Brasil. Atlântica 12(1):5-17.

Andreata, J. V.; Maurer, B. C.; Baptista, M. G. S.; Manzano, F. V.; Teixeira, D. E.; Longo, M. M. \& Ferret, N. V. 2002. Composição da assembléia de peixes da Baía da Ribeira, Angra dos Reis, Rio de Janeiro, Brasil. Revista Brasileira de Zoologia 19(4):1139-1146.

Araújo, F. G. \& DE Azevedo, M. C. C. 2001. Assemblages of southeast-south Brazilian coastal systems as indicated by fishes distribution. Estuarine, Coastal and Shelf Science 52:729-738.

Barbosa, S. R. C. S. \& Begossi, A. 2004. Fisheries, Gender and Local Changes at Itaipu Beach, Rio de Janeiro, Brazil: an individual approach. Multiciência 2:1-13.

Begossi, A. 2006. Temporal stability in fishing spots: conservation and co-management in Brazilian artisanal fisheries. Ecology and Society 11(1):5. Disponível em: <http://www.ecologyandsociety.org/vol11/iss $1 /$ art $5 /$ /. Acesso em: 31.05.2007.

Blaber, S. J. M. 2002. 'Fish in hot water': the challenges facing fish and fisheries research in tropical estuaries. Journal of Fish Biology 61(Supplement A):1-20.

Boesch, D. F. 1977. Application of numerical classification in ecological investigations of water pollution. Corvallis, Environmental Protection Agency. 114p.

Briggs, J. C. 1974. Marine Zoogeography. New York, McGrawHill. 475p.

Carvalho-Filho, A. 1999. Peixes: costa brasileira. São Paulo, Melro. 320p.
Chao, L. N. \& Musick, J. A. 1977. Life history, feeding habits, and functional morphology of juvenile sciaenid fishes in the York River estuary, Virginia. Fishery Bulletin 75:657-702.

Chao, L. N.; Pereira, L. E.; Vieira, J. P.; Bemvenuti, M. A. \& Cunha, L. P. R. 1982. Relação preliminar dos peixes estuarinos e marinhos da lagoa dos Patos e região costeira adjacente, Rio Grande do Sul, Brasil. Atlântica 5:67-75.

Félix, F. C.; Spach, H. L.; Moro, P. S.; Schwarz Jr., R.; Santos, C.; Hackradt, C. W. \& Hostim-Silva, M. 2007. Utilization patterns of surf zone inhabiting fish from beaches in Southern Brazil. Pan-American Journal of Aquatic Sciences 2(1):27-39.

Ferreira, C. E. L.; Gonçalves, J. E. A. \& Coutinho, R. 2001. Community structure of fishes and habitat complexity on a tropical rocky shore. Environmental Biology of Fishes 61:353-369.

Figueiredo, J. L. 1977. Manual de peixes marinhos do sudeste do Brasil. I. Introdução. Cações, raias e quimeras. São Paulo, MZUSP. 104p.

Figueiredo, J. L. \& Menezes, N. A. 1978. Manual de peixes marinhos do sudeste do Brasil. II. Teleostei (1). São Paulo, MZUSP. 110p

1980. Manual de peixes marinhos do sudeste do Brasil. III. Teleostei (2). São Paulo, MZUSP. 90p.

2000. Manual de peixes marinhos do sudeste do Brasil. VI. Teleostei (5). São Paulo, MZUSP. 116p.

Floeter, S. R.; Guimarães, R. Z. P.; Rocha, L. A.; Ferreira, C. E. L.; Rangel, C. A. \& Gasparini, J. L. 2001. Geographic variation in reef-fish assemblages along the Brazilian coast. Global Ecology \& Biogeography 10:423-431.

Floeter, S. R.; Krohling, W.; Gasparini, J. L.; Ferreira, C. E. L. \& ZALMON, I. R. 2007. Reef fish community structure on coastal islands of the southeastern Brazil: the influence of exposure and benthic cover. Environmental Biology of Fishes 78: $147-160$

Friedlander, A.; Sladek-Nowlis, J.; Sanchez, J. A.; Appeldoorn, R.; Usseglio, P.; McCormick, C.; Bejarano, S. \& Mitchell-Chui, A. 2003. Designing effective marine protected areas in Seaflower Biosphere Reserve, Colômbia, base on biological and sociological information. Conservation Biology 17(6): 1769-1784.

Gratwicke, B; Petrovic, C. \& Speight, M. R. 2006. Fish distribution and ontogenetic habitat preferences in non-estuarine lagoons and adjacent reefs. Environmental Biology of fishes 76: $191-210$.

Gray, C. A. \& Kennelly, S. J. 2003. Catch characteristics of the commercial beach-seine fisheries in two Australian barrier estuaries. Fisheries Reasearch 63:405-422.

Humann, P. \& Deloach, N. 2002. Reef fish identification. Florida, Caribbean, Bahamas. Jacksonville, New World. 481p.

Kant de Lima, R. \& Pereira, L. F. 1997. Pescadores de Itaipu: meio ambiente, conflito e ritual no litoral do Estado do Rio de Janeiro. Niterói, EDUFF. 331p.

Lamberth, S. J.; Sauer, W. H. H.; Mann, B. Q.; Brower, S. L.; Clarck, B. M. \& Erasmus, C. 1997. The status of the South African beach-seine and gill-net fisheries. South African Journal of Marine Science 18:195-202.

Laymann, C. A. 2000. Fish assemblage structure of the shallow ocean surf-zone on the eastern shore of Virginia barrier islands. Estuarine Coastal and Shelf Science 51(2): 201-213.

Levinton, J. S. 1982. Marine Ecology. Englewood Cliffs, Prentice-Hall Inc. 526p.

Livingston, R. J. 2002. Trophic organization in costal systems. Boca Raton, CRC. 388p.

Menezes, N. A. \& Figueiredo, J. L. 1980. Manual de peixes marinhos do sudeste do Brasil. IV. Teleostei (3). São Paulo, MZUSP. 96p.

1985. Manual de peixes marinhos do sudeste do Brasil. V. Teleostei (4). São Paulo, MZUSP. 105p.

Methyen, D. A.; Haedrich, R. L. \& Rose, G. A. 2001. The fish assemblage of a Newfoundland estuary: diel, monthly and annual variation. Estuarine, Coastal and Shelf Science 52:669-687.

Milligan, G. \& Cooper, M. 1987. Methodology review: clustering methods. Applied Psychological Measurement 11:329-354.

Monteiro-Neto, C.; Blacher, C.; Laurent, A. S.; Snizek, F. N.; Canozzi, M. B. \& Tabajara, L. L. C. 1990. Estrutura da 
comunidade de peixes de águas rasas na região de Laguna, Santa Catarina, Brasil. Atlântica 12(2):53-69.

Monteiro-Neto, C.; Cunha, L. P. R. \& Musick, J. A. 2003. Community structure of surf-zone fishes at Cassino beach, Rio Grande do Sul, Brazil. Journal of Coastal Research. SI. Brazilian Sandy Beach 35:492-501.

Murray, S. N.; Ambrose, R. F.; Bohnsack, J. A.; Botsford, L.W.; Carr, M. H.; Davis, G. E.; Dayton, P. K.; Gotshall, D.; Gunderson, D. R.; Hixon, M. A.; Lubchenco, J.; Mangel, M.; MacCall, A.; McArdle, D. A.; Ogden, J. C.; Roughgarden, J.; Starr, R. M.; Tegner, M. J. \& Yoklavich, M. M. 1999. Notake reserve networks: sustaining fishery populations and marine ecosystems. Fisheries 24:11-25.

Musick, J. A.; Colovocoresses, J. A. \& Foell, E. J. 1985. Seasonality and the distribution, availability and composition of fish assemblages in Chesapeake bighth. In: YÁñEs-AranciBIA, A. ed. Fish Community Ecology in Estuaries and Coastal Lagoons: Towards an Ecosystem Integration. México, UNAM. p.451-474.

Nagelkerken, I.; Dorenbosch, M.; Verberk, W. C. E. P.; Cocheret de LA Morinière, E. \& VAn der Velde, G. 2000. Importance of shallow-water biotopes of a Caribbean bay for juvenile coral reef fishes: patterns in biotope association, community structure and spatial distribution. Marine Ecology Progress Series 202: 175-192.

Nero, V. L. \& Sealey, K. S. 2006. Fish-environment associations in the coastal waters of Andros Island, The Bahamas. Environmental Biology of Fishes 75:223-236.
Paiva-Filho, A. M.; Giannini, R.; Ribeiro-Neto, F. B. \& Schmiegelow, J. M. M. 1987. Ictiofauna do complexo baía-estuário de Santos e São Vicente, SP, Brasil. Relatório Interno do Instituto Oceanográfico da Universidade de São Paulo 17: $1-10$.

Pessanha, E. G. Da F. 2003. Os companheiros. Trabalho e sociabilidade na pesca em Itaipu. Niterói, EDUFF. 156p.

Rangel, C. A.; Monteiro-Neto, C.; Ferreira, C. E. L. \& Luiz-Júnior, O. J. 2005. Escondidos nos Recifes. Ciência Hoje 36(216):66-68

Salvador, M. V. S. \& Silva, M. A. M. 2002. Morphology and sedimentology of Itaipu embayament - Niterói/RJ. Anais da Academia Brasileira de Ciências 74(1):127-134.

Sergipense, S. \& Pinto, D. G. 1995. Aspectos de ocorrência e distribuição espacial da ictiofauna da lagoa de Itaipu, Niterói, Rio de Janeiro. Boletim do Instituto Oceanográfico da Universidade de São Paulo 11:179-186.

Statsoft Inc. 2006. Electronic Statistics Textbook. Disponível em: <http://www.statsoft.com/textbook/stathome.html>. Acesso em: 08.01.2005.

Stoner, A. W. 2006. What constitutes essential nursery habitat for a marine species? A case study of habitat form and function for queen conch. Marine Ecology Progress Series 257:275-289.

Vieira, J. P. \& Musick, J. A. 1993. Latitudinal patterns in diversity of fishes in warn-temperate and tropical estuarine waters of the western Atlantic. Atlântica 5:115-133.

Wassermann, J. C. \& Alves, A. R. 2004. O holismo aplicado ao conhecimento ambiental. Engevista 6(3):113-120.

Recebido em fevereiro de 2006. Aceito em agosto de 2007. ISSN 0073-4721

Artigo disponível em: www.scielo.br/isz 